\title{
Bistability of Anderson localized states in nonlinear random media
}

\author{
Ilya V. Shadrivov, ${ }^{1}$ Konstantin Y. Bliokh, ${ }^{1,2}$ Yuri S. Kivshar, ${ }^{1}$ Yuri P. Bliokh, ${ }^{3}$ and Valentin Freilikher ${ }^{4}$ \\ ${ }^{1}$ Nonlinear Physics Center, Research School of Physics and Engineering, \\ Australian National University, Canberra ACT 0200, Australia \\ ${ }^{2}$ Applied Optics Group, School of Physics, National University of Ireland, Galway, Galway, Ireland \\ ${ }^{3}$ Department of Physics, Technion-Israel Institute of Technology, Haifa 32000, Israel \\ ${ }^{4}$ Department of Physics, Bar-Ilan University, Ramat-Gan 52900, Israel
}

\begin{abstract}
We study wave transmission through one-dimensional random nonlinear structures and predict a novel effect resulting from an interplay of nonlinearity and disorder. We reveal that, while weak nonlinearity does not change the typical exponentially small transmission in the regime of the Anderson localization, it affects dramatically the disorder-induced localized states excited inside the medium leading to bistable and nonreciprocal resonant transmission. Our numerical modelling shows an excellent agreement with theoretical predictions based on the concept of a high-Q resonator associated with each localized state. This offers a new way for all-optical light control employing statistically-homogeneous random media without regular cavities.

PACS numbers: 42.25.Dd, 42.65.Pc, 05.60.Gg
\end{abstract}

The localization of waves in disordered media, also known as Anderson localization, is a universal phenomenon predicted and observed in a variety of classical and quantum wave systems [1]. Recent renewed interest to this phenomenon is driven by a series of experimental demonstrations in optics [2] and Bose-Einstein condensates [3]. One of the important issues risen in these studies is that the disordered systems can be inherently nonlinear, so that an intriguing interplay of nonlinearity and disorder could be studied experimentally.

Nonlinear interaction between the propagating waves and disorder can significantly change the interference effects, thus fundamentally affecting localization [4, 5]. However, most of the studies of the localization in random nonlinear media deal with the ensemble-averaged characteristics of the field, such as the mean field and intensity, correlation functions, etc. These quantities describe the averaged, typical behavior of the field, but they do not contain information about individual localized modes (resonances), which exist in the localized regime in each realization of the random sample [6 9 ]. These modes are randomly located in both real space and frequency domain and are associated with the exponential concentration of energy and resonant tunnelling. In contrast to regular resonant cavities, the Anderson modes occur in a statistically-homogeneous media because of the interference of the multiply scattered random fields. Although the disorder-induced resonances in linear random samples have been the subject of studies for decades, the resonant properties of nonlinear disordered media have not been explored so far.

In this Letter we study the effect of nonlinearity on the Anderson localized states in a one-dimensional random medium. As a result of interplay of nonlinearity and disorder, bistability and nonreciprocity appear upon resonant wave tunnelling and excitation of disorder-induced localized modes in a manner similar to that for regular cavity modes. At the same time, weak nonlinearity has practically no effect on the averaged localization background.

First, we consider a stationary problem of the transmission of a monochromatic wave through a onedimensional random medium with Kerr nonlinearity. The problem can be reduced to the equation

$$
\frac{d^{2} \psi}{d x^{2}}+k^{2}\left[n^{2}-\chi|\psi|^{2}\right] \psi=0
$$

where $\psi$ is wave field, $x$ is coordinate, $k$ is wave number in the vacuum, $n=n(x)$ is the refractive index of the medium, and $-\chi$ is the Kerr coefficient.

In the linear regime, $\chi|\psi|^{2}=0$, the multiple scattering of the wave on the random inhomogeneity $n^{2}(x)$ brings about Anderson localization. The main signature of the localization is an exponential decay of the wave intensity, $I=|\psi|^{2}$, deep into the sample and, thus, an exponentially small transmission [1, 10]: $I_{\text {out }}^{(\text {typ })} \sim$ $I_{\text {in }} \exp \left(-2 L / l_{\text {loc }}\right) \ll 1$. Here $L$ is the length of the sample and $l_{\text {loc }}$ is the localization length which is the only spatial scale of Anderson localization. Along with the typical wave transmission, there is an anomalous, resonant transmission, which accompanies excitation of the Anderson localized states inside the sample and occurs at random resonant wave numbers $k=k_{\text {res } 0}$ [6 6 ]. In this case, the intensity distribution in the sample is characterized by an exponentially localized high-intensity peak inside the sample, $I_{\text {peak }} \gg I_{\text {in }}$, and a transmittance much higher than the typical one: $I_{\text {out }}^{(\text {res })} \gg I_{\text {out }}^{(\text {typ })}$ (see Fig. 1).

Excitation of each localized mode inside the random sample can be associated with an effective resonator cavity located in the area of field localization and characterized by high quality factor $Q \gg 1$ [11]. According to this model, the transmittance spectrum $T(k)$ in the vicinity of a resonant wavelength, $\left|k-k_{\text {reso } 0}\right| \ll k_{\text {res0 }}$ is given by 


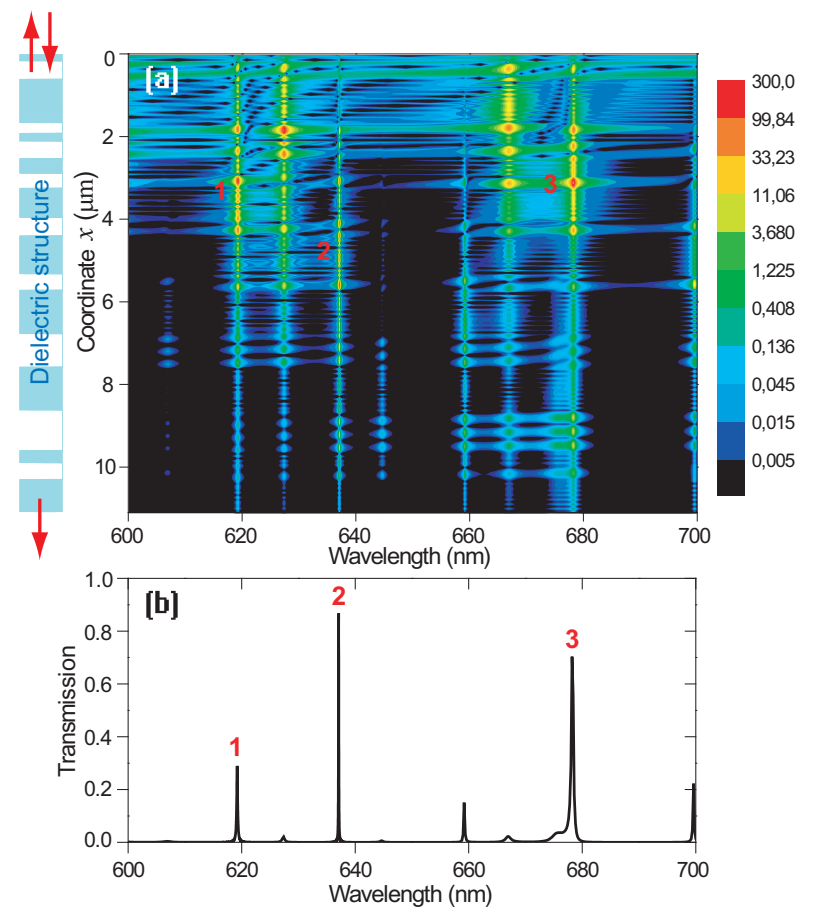

FIG. 1: (Color online.) Excitation of localized modes in the linear regime: (a) normalized intensity of the field, $I / I_{\text {in }}$ (log scale), vs. the wavelength and position in the sample and (b) the transmission spectrum.

the Lorentzian dependence [7, 12]:

$$
T(k)=\frac{T_{\mathrm{res}}}{1+\left[2 Q\left(k / k_{\mathrm{res} 0}-1\right)\right]^{2}}
$$

where $T_{\text {res }}=T\left(k_{\text {res } 0}\right)$.

Let us account now for the effect of weak nonlinearity: $\left|\chi \psi^{2}\right| \ll 1$. The nonlinearity becomes noticeable, first of all, at the resonant intensity peaks $I_{\text {peak }}$ inside the sample. It is physically clear that the Kerr term in Eq. (1) changes the effective refractive index of the medium leading to the intensity-dependent shift of the resonant wave number: $k_{\text {res } 0} \rightarrow k_{\text {res }}\left(I_{\text {peak }}\right)$. Since the values of $I_{\text {peak }}$ and $I_{\text {out }}$ are unambiguously connected, the resonant wave number is a function of the output intensity, which in the case of weak nonlinearity takes the form:

$$
k_{\text {res }}\left(I_{\text {out }}\right) \simeq k_{\text {res } 0}+\left.\frac{d k_{\text {res }}}{d I_{\text {out }}}\right|_{I_{\text {out }}=0} I_{\text {out }} .
$$

Substitution Eq. (3) into Eq. (2) yields

$$
T\left(k, I_{\text {out }}\right) \equiv \frac{I_{\text {out }}}{I_{\text {in }}}=\frac{T_{\text {res }}}{1+\left[A \chi I_{\text {out }}+\delta\right]^{2}} .
$$

Here we introduced two dimensionless parameters $A$ and $\delta$, which characterize, respectively, the strength of the nonlinear feedback and the detuning from the resonant wave number:

$$
A=\left.\frac{2 Q}{\chi} \frac{d \ln k_{\mathrm{res}}}{d I_{\mathrm{out}}}\right|_{I_{\mathrm{out}}=0}, \delta=2 Q\left(1-\frac{k}{k_{\mathrm{res} 0}}\right) .
$$

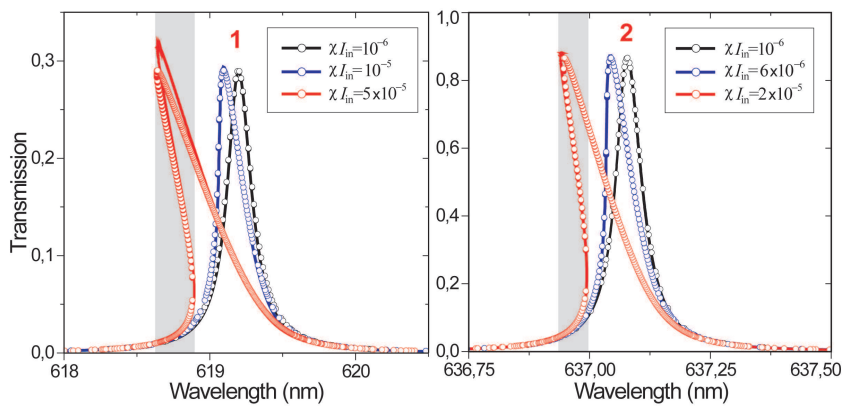

FIG. 2: (Color online.) Nonlinear deformations of the transmission spectra of the resonances 1 and 2 from Fig. 1 at different intensities of the incident wave: numerical simulations of Eq. (1) (curves) and theoretical Eq. (4) (symbols). Light-grey stripes indicate three-valued regions for the highintensity curves, where only two of them (corresponding to the lower and upper branches) are stable.

Equation (44) establishes relation between the input and output wave intensities, which is cubic with respect to $I_{\text {out }}$. It has a universal form typical for nonlinear resonators possessing optical bistability [13]. From Eq. (5) it follows that in the region of parameters:

$$
A \delta<0, \quad \delta^{2}>3, \quad|\chi| I_{\text {in }}>\frac{8}{3 \sqrt{3}} \frac{1}{|A| T_{\text {res }}},
$$

the dependence $I_{\text {out }}\left(I_{\text {in }}\right)$ is of the S-type and the stationary transmission spectrum $T(k)$ is a three-valued function. In most cases, one of the solutions is unstable, whereas the other two form a hysteresis loop in the $I_{\text {out }}\left(I_{\text {in }}\right)$ dependence (see Figs. 2 and 3 ).

It is important to emphasize two features of the equations (44) and (5), describing the nonlinear resonant transmission through a localized state. First, they have been derived without any approximations apart from the natural smallness of the nonlinearity and Lorentzian shape of the spectral line. Second, although the resonant transmission, the effect of nonlinearity, and bistability owe their origin to the excitation of the Anderson localized mode inside the sample, equations (4) and (5) contain only quantities which can be found via outside measurements. Indeed, $T_{\text {res }}, k_{\text {res } 0}$, and $Q$ are determined from the transmission spectrum in the linear regime, Eq. (2), while the derivative $d \ln k_{\text {res }} /\left.d I_{\text {out }}\right|_{I_{\text {out }}=0}$ can be retrieved from the shift of the spectral line when the intensity is changed. This enables one to obtain the whole dependence $I_{\text {out }}\left(I_{\mathrm{in}}, k\right)$ for any given resonance performing external measurements of $T(k)$ at only two different intensities of the incident wave.

To verify theoretical predictions, we numerically model the transmission of light incident from $x=0$ through a random sample consisting of $N=19$ alternating layers with dielectric constants $n_{1}^{2}=1$ and $n_{2}^{2}=10$ and random widths uniformly distributed in the range $(0.12,1.08) \mu \mathrm{m}$, Fig. 1. This corresponds to $l_{\mathrm{loc}}=3.53 \mu \mathrm{m}$. Figure 2 
shows nonlinear deformations of the resonant transmission spectra $T(k)$ for different values of $\chi I_{\text {in }}>0$, which exhibit transitions to bistability. The analytical dependence $T(k)$ given by Eqs. (4) and (5) with the parameters $T_{\text {res }}, Q$, and $A$ found from the numerical experiments are in excellent agreement with the direct numerical solutions of Eq. (1). In numerical simulations of stationary regime we used the standard 4-th order Runge-Kutta method. We note, that the incident field amplitude is a singlevalued function of the transmitted field. Thus, we solve second-order ordinary differential equation Eq. (1) using transmitted field as the initial condition for the equation.

The dimensionless parameters $T_{\text {res }}$, and $Q$ from Eqs. (4) 5), can also be estimated from a simple resonator model of the Anderson localized states [7, 8, 11]:

$$
T_{\mathrm{res}}=\frac{4 T_{1} T_{2}}{\left(T_{1}+T_{2}\right)^{2}}, \quad Q^{-1} \sim \frac{T_{1}+T_{2}}{4 k_{\mathrm{res} 0} l_{\mathrm{loc}}}
$$

where

$$
T_{1} \sim \exp \left[-2 x_{\mathrm{res}} / l_{\mathrm{loc}}\right], \quad T_{2} \sim \exp \left[-2\left(L-x_{\mathrm{res}}\right) / l_{\mathrm{loc}}\right]
$$

are the transmission coefficients of the two barriers that form the effective resonator, $x_{\text {res }}$ is the coordinate of the center of the area of field localization, $l_{\text {loc }}$ is the localization length, and $L$ is the length of the sample.

Introducing a weak Kerr nonlinearity into the resonator model, one can also estimate the nonlinear feedback parameter $A$ :

$$
A \sim Q / T_{2} \overline{n^{2}}
$$

where $\overline{n^{2}}$ is the mean value of $n^{2}(x)$.

It is important to note that each disorder-induced resonance is associated with its own effective cavity, so that the disordered sample can be considered as a chain of randomly located coupled resonators [14].

Equations (7)-(9) enable one to estimate the values of the parameters describing the nonlinear resonant wave tunnelling in Eqs. (4) and (5) by knowing only the basic parameters of the localization - the localization coordinate and the localization length. In particular, substituting Eqs. (7)-(9) into Eq. (6) and taking into account that the most pronounced transmission peaks correspond to the localized states with $x \simeq L / 2$ and $T_{1} \sim T_{2}$, we estimate the incident power needed for bistability of localized states:

$$
|\chi| I_{\mathrm{in}} \gtrsim \frac{\exp \left(-2 L / l_{\mathrm{loc}}\right)}{k_{\mathrm{res} 0} l_{\mathrm{loc}}} .
$$

For the parameters used in our simulations this gives quite reasonable value $|\chi| I_{\text {in }} \gtrsim 10^{-5}$. If we increase the length of the sample, the Q-factors of the resonances grow, and the incident power needed to observe bistability becomes smaller.
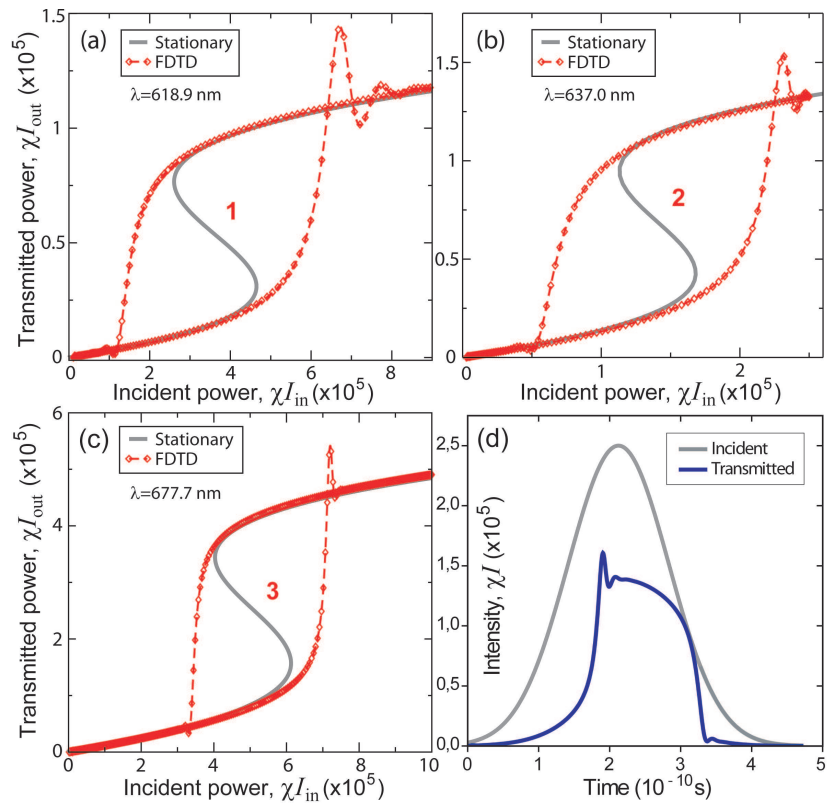

FIG. 3: (Color online.) Stationary and FDTD simulations showing hysteresis loops in the output vs. input power dependence for resonances 1,2, and 3 in Fig. 1 Panel (d) shows deformation of the transmitted Gaussian pulse corresponding to the hysteresis switching on the resonance 2 .

To demonstrate temporal dynamics upon the bistable resonant tunnelling, we implemented an explicit iterative nonlinear finite-difference time-domain (FDTD) scheme for modelling pulse propagation through the disordered nonlinear sample. For precise modelling of the spectra of narrow high-Q resonances, we employed fourth-order accurate algorithm, both in space and in time [15], as well as the Mur boundary conditions to simulate open boundaries and total-field/scattered-field technique for exciting the incident wave. Sufficient accuracy was achieved by creating a dense spatial mesh of 300 points per wavelength $(d x=\lambda / 300)$. To assure stability of the method in nonlinear regime, we used the time step of $d t=d x / 3 c$, and each simulation ran for $N=2 * 10^{8}$ time steps. To compare the results of the FDTD simulations with the steady-state theory, we consider transmission of long Gaussian pulses with central frequencies and amplitudes satisfying conditions (10), see Fig. 3(d). With an appropriate choice of the signal frequencies, we observe hysteresis loops in the $I_{\text {out }}\left(I_{\text {in }}\right)$ dependences which are in excellent agreement with stationary calculations, as shown in Figs. 3 $(a-c)$. Transitional oscillations typical for bistable nonlinear structure accompany jumps between two stable branches [16], and strong reshaping of the transmitted pulse evidences switching between the two regimes of transmission, Fig. 3(d). Period of the transitional oscillations is defined by mismatch between external wave frequency and nonlinear eigenfrequency, whereas the decay rate is defined by the $\mathrm{Q}$-factor. We note, that dif- 


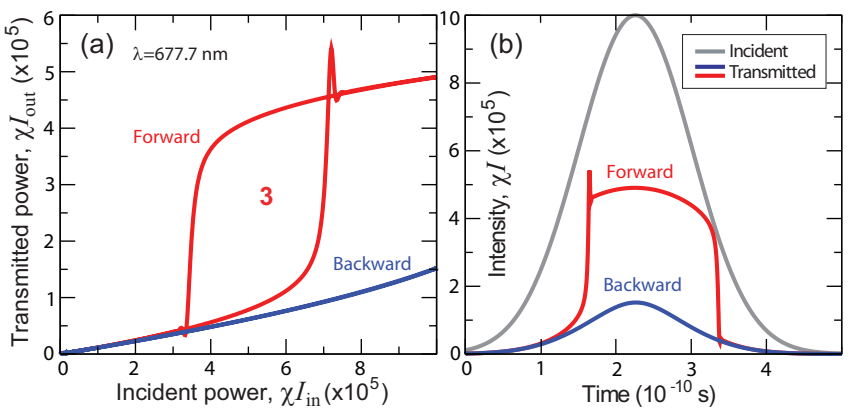

FIG. 4: (Color online.) (a) Non-reciprocal transmission through the nonlinear disordered structure, showing different output powers for identical waves incident from different directions. (b) Corresponding shape of the incident pulse, and pulses transmitted in different directions.

ferent choice of the signal frequencies near the resonance can lead to various other behaviours of output vs. input curves, with transmission either increasing, when nonlinear resonance frequency shifts towards the signal frequency, or decreasing in the opposite case.

In addition to bistability, the resonant wave tunnelling through a nonlinear disordered structure is nonreciprocical. As is known for regular systems, nonsymmetric and nonlinear systems may possess nonreciprocal transmission properties, resembling the operation of a diode. An all-optical diode is a device that allows unidirectional propagation of a signal at a given wavelength, which may become useful for many applications [16, 17]. A disordered structure is naturally asymmetric in the generic case, and one may expect a nonreciprocal resonant transmission in the nonlinear case. To demonstrate this, we modelled propagation of an electromagnetic pulse impinging the same sample from different sides and monitor the transmission characteristics. One case of such nonreciprocical resonant transmission is shown in Fig. 4. We observe considerably different transmission properties in opposite directions with the maximal intensity contrast between two directions 7.5:1. Moreover, the threshold of bistability is also significantly different for two directions: there is a range of incident powers, for which the wave incident from one side of the sample is bistable, while there is no signs of bistability for the incidence from the other side. Figure 4(b) shows the pulse reshaping for incidence from opposite sides of the structure.

To conclude, we have studied the wave transmission through a weakly-nonlinear statistically homogeneous one-dimensional random medium and demonstrated novel manifestations of the interplay between nonlinearity and disorder. We have shown that even weak nonlinearity affect dramatically the resonant transmission associated with the excitation of the Anderson localized states leading to bistability and nonreciprocity. Despite random character of the appearance of Anderson modes, their behavior and evolution are rather de- terministic, and, therefore, these modes can be used for efficient control of light similar to regular cavity modes. Numerical modelling shows an excellent agreement with theoretical analysis based on the concept of a high-Q resonator associated with each localized state. Our results demonstrate that, unlike infinite systems, the Anderson localization in finite samples is not destroyed by weak nonlinearity - instead it exhibits new intriguing features typical for resonant nonlinear systems.

The authors acknowledge a support of the Australian Research Council (Linkage International and Discovery projects), European Commission (Marie Curie fellowship), and the Science Foundation Ireland.

[1] P.W. Anderson, Phys. Rev. 109, 1492 (1958); P. Sheng, Scattering and Localization of Classical Waves in Random Media (World Scientific, Singapore, 1990).

[2] J. Bertolotti et al., Phys. Rev. Lett. 94, 113903 (2005); J. Topolancik, B. Ilic, and F. Vollmer Phys. Rev. Lett. 99, 253901 (2007); T. Schwartz et al., Nature 446, 52 (2007); Y.Lahini et al., Phys. Rev. Lett. 100, 013906 (2008).

[3] D. Clément et al., Phys. Rev. Lett. 95, 170409 (2005); T. Schulte et al., Phys. Rev. Lett. 95, 170411 (2005); J. Billy et al., Nature 453, 891 (2008); G. Roati et al., Nature 453, 895 (2008).

[4] S.A. Gredeskul and Y.S. Kivshar, Phys. Rep. 216, 1 (1991); J. D. Maynard, Rev. Mod. Phys. 73, 401 (2001).

[5] P. Devillard and B. Souillard, J. Stat. Phys. 43, 423 (1986); B. Doucot and R. Rammal, Europhys. Lett. 3, 969 (1987); Y.S. Kivshar et al., Phys. Rev. Lett. 64, 1693 (1990); M.I. Molina and G.P. Tsironis, Phys. Rev. Lett. 73, 464 (1994); V.A. Hopkins et al., Phys. Rev. Lett. 76, 1102 (1996); G. Kopidakis and S. Aubry, Phys. Rev. Lett. 84, 3236 (2000); A.S. Pikovsky and D.L. Shepelyansky, Phys. Rev. Lett. 100, 094101 (2008).

[6] U. Frisch et al., Phys. Rev. A 8, 1416 (1973); M.Y. Azbel and P. Soven, Phys. Rev. B 27, 831 (1983); M.Y. Azbel, Phys. Rev. B 28, 4106 (1983).

[7] K.Y. Bliokh, Y.P. Bliokh, and V.D. Freilikher, J. Opt. Soc. Am. B 21, 113 (2004).

[8] K.Y. Bliokh et al., Phys. Rev. Lett. 97, 243904 (2006).

[9] J. Topolancik, F. Vollmer, and B. Ilic, Appl. Phys. Lett. 91, 201102 (2007).

[10] M.V. Berry and S. Klein, Eur. J. Phys. 18, 222 (1997).

[11] K.Y. Bliokh et al., Rev. Mod. Phys. 80, 1201 (2008).

[12] P. Sebbah et al., Phys. Rev. Lett. 96, 183902 (2006).

[13] E. Abraham and S.D. Smith, Rep. Prog. Phys. 45, 815 (1982); H.M. Gibbs, S.L. McCall, and T.N.C. Venkatesan, Phys. Rev. Lett. 36, 1135 (1976); F.S.Felber and J.H. Marburger, Appl. Phys. Lett. 28, 731 (1976); M. Soljačić et al., Phys. Rev. E 66, 055601(R) (2002); M. Notomi et al., Opt. Express 13, 2678 (2005).

[14] K.Y. Bliokh et al., Phys. Rev. Lett. 101, 133901 (2008).

[15] K.-P. Hwang, IEEE Microwave and Wireless Components Lett. 15, 271 (2005).

[16] M.W. Feise et al., Phys. Rev. E 71, 037602 (2005).

[17] M.D. Tocci et al., Appl. Phys. Lett. 66, 2324 (1995); J.J. Liang et al., Appl. Phys. Lett. 70, 1192 (1997); K. Gallo and G. Assanto, J. Opt. Soc. Am. B 16, 267 (1999). 